\title{
A PRACTICAL STATISTICAL APPROACH TO THE RECONSTRUCTION PROBLEM USING A SINGLE SLICE REBINNING METHOD
}

\author{
Robert Cierniak $^{1, *}$, Piotr Pluta ${ }^{1}$, Andrzej Kaźmierczak $^{2,3}$ \\ ${ }^{1}$ Institute of Computational Intelligence, Czestochowa University of Technology, \\ al. Armii Krajowej 36, 42-200 Czesstochowa, Poland \\ ${ }^{2}$ Information Technology Institute \\ University of Social Sciences, 90-113 Łódź \\ ${ }^{3}$ Clark University Worcester, MA 01610, USA \\ ${ }^{*}$ E-mail: robert.cierniak@pcz.pl
}

Submitted: 11th September 2019; Accepted: 5th March 2020

\begin{abstract}
The paper presented here describes a new practical approach to the reconstruction problem applied to 3D spiral $\mathrm{x}$-ray tomography. The concept we propose is based on a continuous-to-continuous data model, and the reconstruction problem is formulated as a shift invariant system. This original reconstruction method is formulated taking into consideration the statistical properties of signals obtained by the 3D geometry of a CT scanner. It belongs to the class of nutating reconstruction methods and is based on the advanced single slice rebinning (ASSR) methodology. The concept shown here significantly improves the quality of the images obtained after reconstruction and decreases the complexity of the reconstruction problem in comparison with other approaches. Computer simulations have been performed, which prove that the reconstruction algorithm described here does indeed significantly outperforms conventional analytical methods in the quality of the images obtained.
\end{abstract}

Keywords: reconstruction algorithm, statistical iterative method, computed tomography

\section{Introduction}

Even though computed tomography was introduced in clinical practice many years ago, it continues to be a very attractive field of research. Every new generation of CT device stimulates the development of reconstruction algorithms adapted for the new scanner design. It can be said that all the most significant reconstruction algorithms belong to one of two categories: those methodologies which are based on a discrete-to-discrete (D-D) data model and those based on a continuous-to-continuous (C-
C) data model, especially those based on a convolution and back-projection (FBP) strategy $[1,2]$. The use of algebraic reconstruction algorithms, (i.e. the algebraic reconstruction technique ART, which belongs to the D-D class) in the first historical CT apparatus was presumably because there was no real alternative at the time. After this "early mistake", the next generation of CT systems used only reconstruction algorithms based on FBP image processing methods. The main reason for this was the huge size of the matrices which appear in the algebraic reconstruction problem and the calcula- 
tion complexity of the reconstruction method based on this methodology that this caused. The analytical (or transformation) methodology drastically simplifies the number of calculations needed and so is more appealing. Although this situation has changed somewhat, as it has been proven (e.g. [3]) that the frequency of cancerous diseases in patients who had had a CT scan (at least one year after the scan) is about $24 \%$ higher than in the case of patients who had not had the scan. Due to the enormous prevalence of CT scans, any actions aimed at reducing this impact are of fundamental importance, assuming of course, the continued existence of this popular, cheap, and effective diagnostic technique. For these reasons, but also for social and commercial ones, producers of CT scanners began a kind of competition to develop methods of reducing the X-ray dose absorbed by patients. Recently, some commercial solutions of such systems have been developed, which perform reconstruction processing iteratively to decrease the noise in the images. The most interesting approach is a modelbased iterative reconstruction (MBIR) $[4,5]$, where a statistical model of the measurement signals is derived analytically, and, based on this, an iterative reconstruction algorithm is formulated (see also e.g. $[6,7])$. A crucial part in this approach is played by the reconstruction problem formulated according to a D-D scheme. The reconstruction idea presented in the above publications is based on the maximum likelihood (ML) approach and a development of this concept - the maximum a posteriori probability (MAP) estimation approach (the iterative coordinate descent (ICD) algorithm described comprehensively in [8] implements the MAP approach). Consequently, in 2013, this development had its debut under its commercial name Veo CT Model-Based Iterative Reconstruction. The application of this reconstruction method, however, presents some significant technical difficulties in its practical realization, e.g. the difficulty in establishing the coefficients of the forward model for 3D spiral cone-beam scanners $[8,9]$. The huge number of these coefficients in this model means that it is impossible to keep all of them in memory at the same time and the requirement for the simultaneous calculation of all voxels in the range of the reconstructed 3D image makes the reconstruction problem extremely complex. Although there have been attempts to decrease the calculation complex- ity of this approach, as presented, for example, in the paper [10], they have, as yet, only met with limited results. Moreover, this system uses a reconstruction problem model that has been shown to be extremely ill-conditioned [11]. Therefore, there is still room for improvement in such systems. It would be much more profitable to construct a statistical reconstruction method which would take into consideration the statistical conditions of the measurement process and the geometry of the projections performed, as in the ICD algorithm, thereby eliminating most of the disadvantages of the D-D scheme. In previous papers, we have shown how to formulate the $\mathrm{C}-\mathrm{C}$ reconstruction problem consistent with the ML methodology for scanners with parallel geometry [13, 12], for fan-beams [14], and finally we have proposed a scheme of reconstruction method for the spiral cone-beam scanner [15]. Our approach has some significant advantages compared with the D-D methodology. Firstly, in our method, we establish certain coefficients, but this is performed in a much easier way than in comparable methods. Secondly, we perform the reconstruction process in only one plane in 2D space, greatly simplifying the problem. In this way, the reconstruction process can be performed for every cross-section image separately. After this, it is possible to reconstruct the whole 3D volume image from the set of previously reconstructed 2D images. And finally, because of the C-C methodology of the reconstruction process, we can perform most of the computationally expensive operations in the frequency domain (2D convolutions). Because it is a very much less computationally demanding approach, by using FFT, we make our reconstruction method independent of the dimensions of the reconstructed image, to an acceptable degree. This approach also outperforms the D-D method regarding the better condition number at the level of problem formulation [11]. This makes our method more competitive in terms of its resistance to the influence of noise and errors in the forward model, and it opens up a new perspective for the regularization of the reconstruction problem. The main motivation for this paper is to present a feasible, practical solution for 3D spiral tomographic scanners based on the analytical statistical reconstruction approach mentioned above. 


\section{3D Reconstruction Method for the Spiral CT Scanner}

Fundamental for our method, the 2D analytical approximate reconstruction problem was originally formulated for measurements from a scanner with parallel geometry $[12,13]$. Because the proposed approach is strongly associated with the parallel geometry of the scanner, we preferred to choose for further consideration a signal processing procedure from among reconstruction methods related to socalled rebinning methods. This could be, for example, the reconstruction concept involved in the single slice rebinning (SSR) method (see e.g. $[16,17]$ ) or based on the principles of the more advanced approach, i.e. the advanced single slice rebinning (ASSR) algorithm (see e.g. [18, 19]). Because the ASSR method was better from a practical point of view, this latter approach was chosen. In our further analysis, we will present how we adapt this idea using our statistical reconstruction concept. The scheme of the reconstruction procedure we propose is given in Figure 1.

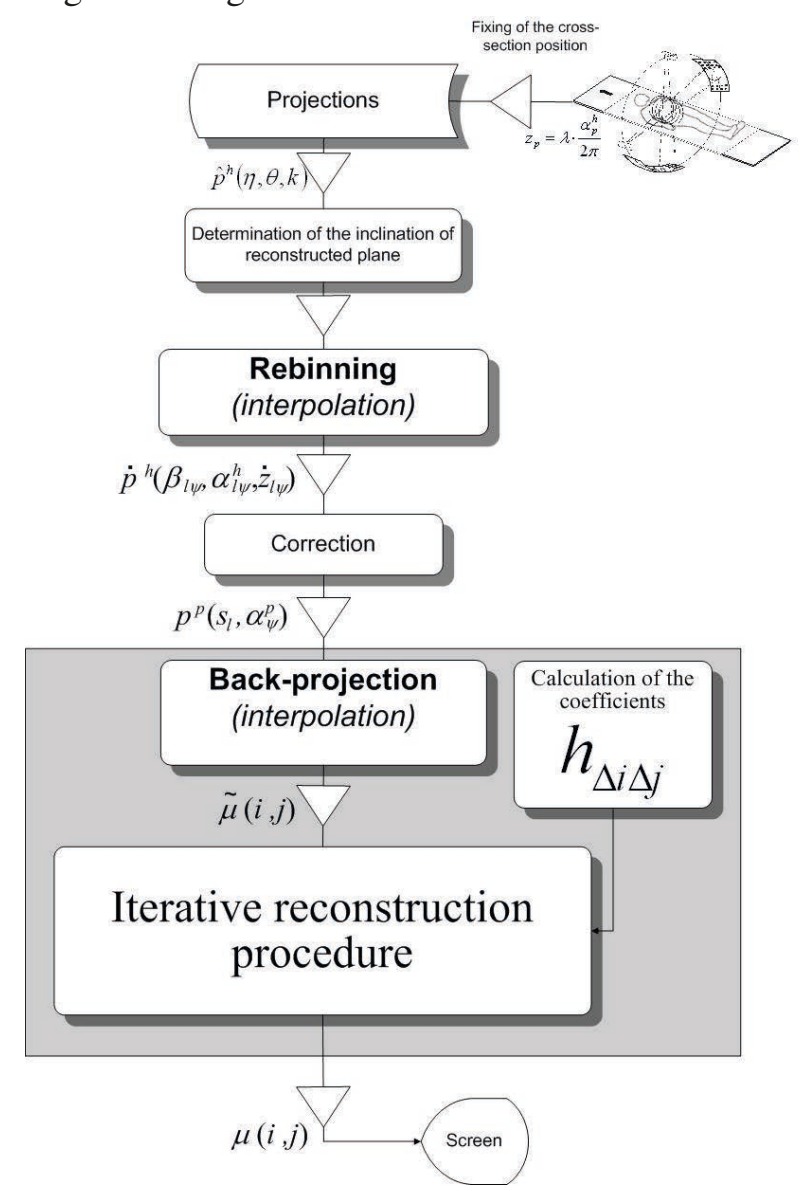

Figure 1. A general scheme of the image reconstruction algorithm for the spiral scanner

\subsection{The spiral cone-beam scanner}

The geometry of the spiral cone-beam scanner with some trigonometric relationships is shown in Figures 2, 3 and 4.

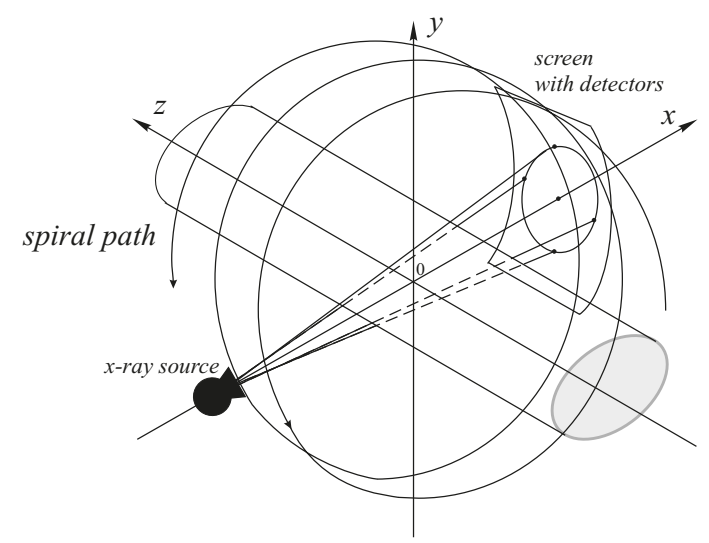

Figure 2. A general 3D view of the projection system

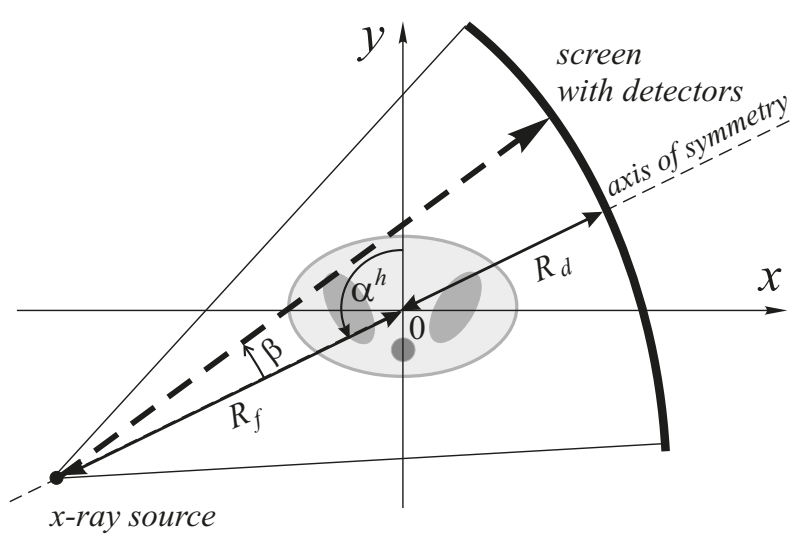

Figure 3. The view in a plane perpendicular to the axis of rotation

$\uparrow y$

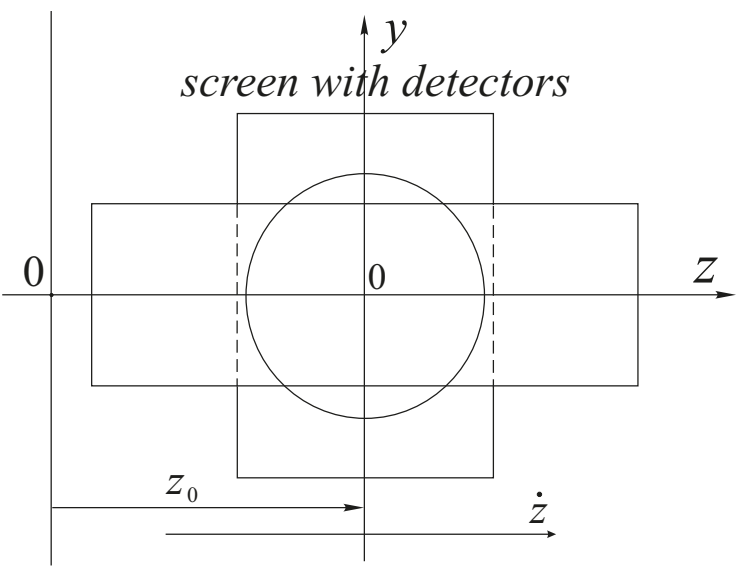

Figure 4. The view in the plane along the axis of rotation of the measurement system

The measurement system comprises an x-ray tube and a partially cylindrically shaped screen with detectors. This assembly rotates around the princi- 
pal axis of the system, i.e. the $z$-axis. At the same time, the table with the patient moves slowly into the gantry. This means that the projection system traces a spiral path around the $z$-axis. Each projection measured can be specified by the following parameters: $p^{h}\left(\beta, \alpha^{h}, \dot{z}\right)$, where $\beta$ is the angle between a particular ray and the axis of symmetry of the measurement system; $\alpha^{h}$ is the angle between the axis of symmetry of the measurement system and the $y$-axis; $\dot{z}$ is the $z$-coordinate relative to the current position of the measurement system.

The focus of the tube moves along the $z$-axis according to the following relation (assuming that the tube starts from $z_{0}=0$ at an angle $\alpha^{h}=0$ )

$$
z_{0}=\lambda \frac{\alpha^{h}}{2 \pi}
$$

where: $\lambda$ is the table increment per full revolution of the tube.

Of course, in a real CT device, the reconstruction algorithm can only make use of projections obtained by a scanner.

Each useful beam reaches an individual detector, and the measurement is specified by its row $k=1,2, \ldots, \mathrm{K}$ in the detector array, where $\mathrm{K}$ is an even number of rows placed on the screen, and its column $\eta=-(\mathrm{H}-1) / 2, \ldots, 0, \ldots,(\mathrm{H}-1) / 2$ in the matrix, where $\mathrm{H}$ is an odd number of detectors in each array. Additionally, projections are only performed at a limited number of angles, each of which is indexed by the variable $\theta=0,1, \ldots$ This discretization can be summarized by saying that the proposed reconstruction algorithm has available to it the following projections values $p^{h}\left(\beta_{\eta}, \alpha_{\theta}^{h}, \dot{z}_{k}\right)$.

\subsection{Inclination of the reconstruction plane}

Before using the algorithm, a doctor needs to determine the location of the middle of the reconstructed slice along the $z$-axis. This position is represented by a value $z_{p}$, and is related to the angle $\alpha_{p}^{h}$ of rotation of the tube at the moment when the center of symmetry of the cone-beam intersects with the reconstruction plane. This can be calculated according to the following equation

$$
\alpha_{p}^{h}=\frac{2 \pi \cdot z_{p}}{\lambda}
$$

After calculating the angle of rotation $\alpha_{p}^{h}$, it is possible to determine the angle of inclination $\gamma$ of the plane of the slice. The approach presented here belongs to the class of so-called "nutating" reconstruction methods (see also [18], [19]), where different reconstruction planes are inclined in different ways, at a specific angle for every position. The main goal of this concept is to adjust the reconstruction plane to the path of the helical movement of the tube around table. The optimal inclination angle meets the following condition

$\gamma=-\arctan \left(\frac{\lambda \cdot \arccos \left(\frac{1}{2}(1+\cos (\pi))\right)}{2 \pi \mathrm{R}_{f} \sin \left(\arccos \left(\frac{1}{2}(1+\cos (\pi))\right)\right)}\right)$,

when errors of interpolation will be minimized.

The conceptional view of this operation is illustrated by Figure 5 .

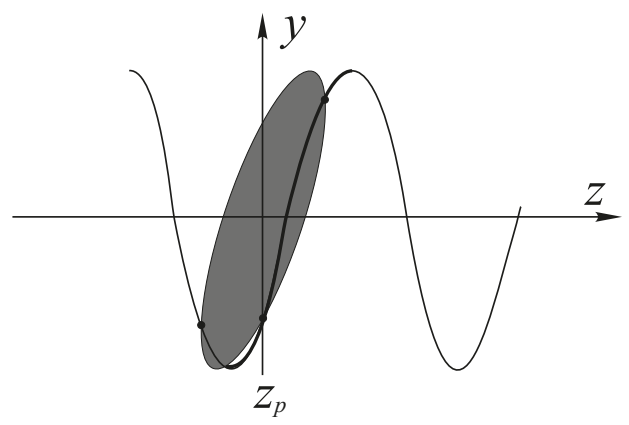

Figure 5. Location of the reconstructed slice

\subsection{Longitudinal approximation}

It is crucial for the presented conception to reformulate the $3 \mathrm{D}$ reconstruction problem in a $2 \mathrm{D}$ problem. Thanks to this it will be possible to perform a reconstruction procedure using parallel virtual rays lying in the reconstruction plane. These virtual parallel rays, which are the projections necessary for the following reconstruction procedure, are approximated in the ASSR method by projections established by interpolation of real measurements performed in scanner. This operation is often called the longitudinal approximation. The positions of both the ray representing the interpolated measurement and the desired parallel ray with respect to the reconstruction plane is shown in Figure 6. 


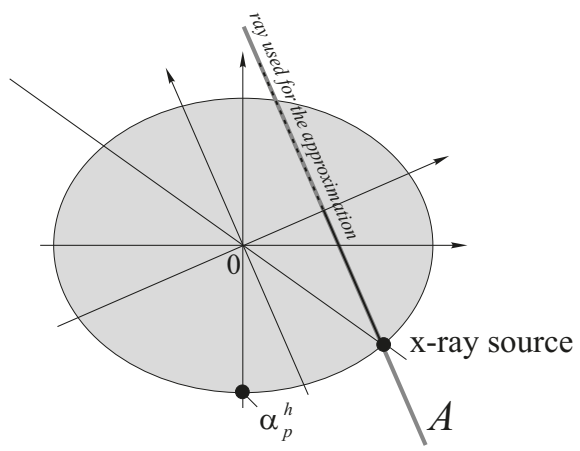

Figure 6. View presenting an idea of the longitudinal approximation

In the longitudinal approximation, we firstly specify all the parameters of the parallel projections $p^{p}\left(s_{l}, \alpha_{\psi}^{p}\right)$ needed for the reconstruction procedure. We assume that the virtual detectors are equidistant on a flat screen and fixed at the points $s_{l}=l \cdot \Delta_{s}$, where $l=-\mathrm{L} / 2-0.5, \ldots, \mathrm{L} / 2-0.5$, and $\mathrm{L}$ is an even number of detectors. Every virtual parallel projection is performed in an equiangular way at specific angles $\alpha_{\psi}^{p}=\psi \cdot \Delta_{\psi}$, where $\psi=0, \ldots, \Psi-1$, and $\Psi$ is the number of projections carried out. Based on the set of values of parameters $\left(s_{l}, \alpha_{\psi}^{p}\right)$, we can establish the projection angles $\alpha^{h}$ at which the helical projections should be performed, according to the following geometrical relation

$$
\alpha_{l \psi}^{h}=\alpha_{\psi}^{p}+\arcsin \frac{s_{l}}{\mathrm{R}_{f}}+\alpha_{p}^{h}
$$

The following pair of equations allows us to calculate the remaining coordinates of this projection onto a cylindrically shaped screen

$$
\beta_{l \psi}=-\arctan \frac{w}{\mathrm{R}_{f}+\mathrm{R}_{d}}
$$

and

$$
\dot{z}_{l \psi}=\frac{v \mathbf{R}_{f d}}{\sqrt{\left(\mathbf{R}_{f}+\mathbf{R}_{d}\right)^{2}+w^{2}}}
$$

where:

$$
w=\frac{\mathrm{R}_{f}+\mathrm{R}_{d}}{\mathrm{R}_{f}} \cdot \frac{s_{l}}{\cos \left(\alpha^{h}-\alpha_{p}^{h}-\alpha_{\psi}^{p}\right)}
$$

and
$v=\frac{\mathrm{R}_{f}+\mathrm{R}_{d}}{\mathrm{R}_{f}}\left(\frac{s_{l} \cdot \cos \left(\alpha^{h}-\alpha_{p}^{h}\right) \cdot \tan \gamma}{\cos \left(\alpha^{h}-\alpha_{p}^{h}-\alpha_{\psi}^{p}\right)}-\lambda \frac{\alpha^{h}-\alpha_{p}^{h}}{2 \pi}\right)$.

It is unlikely that any physical ray will be consistent with the line described by the parameters specified by (4), (5), and (6). That is why an additional interpolation operation is necessary to establish a projection value $\dot{p}^{h}\left(\beta_{l \psi}, \alpha_{l \psi}^{h}, \dot{z}_{l \psi}\right)$. This can be done using trilinear interpolation based on the eight projections nearest to the desired ray

$$
\begin{aligned}
& p_{1, l \psi}^{h}=p^{h}\left(\beta_{\eta_{l \psi}^{\uparrow}}^{\uparrow}, \alpha_{\theta_{l \psi}^{\uparrow}}, \dot{z}_{k_{l \psi}}^{\uparrow}\right) ; \\
& p_{2, l \psi}^{h}=p^{h}\left(\beta_{\eta_{l \psi}}^{\downarrow}, \alpha_{\theta_{l \psi}}^{\uparrow}, \dot{z}_{k_{l \psi}}^{\uparrow}\right) ; \\
& p_{3, l \psi}^{h}=p^{h}\left(\beta_{\eta_{l \psi}^{\uparrow}}^{\uparrow}, \alpha_{\theta_{l \psi}}^{\downarrow}, \dot{z}_{k_{l \psi}}^{\uparrow}\right) \text {; } \\
& p_{4, l \psi}^{h}=p^{h}\left(\beta_{\eta_{l \psi}}^{\downarrow}, \alpha_{\theta_{l \psi}}^{\downarrow}, \dot{z}_{k_{l \psi}}^{\uparrow}\right) ; \\
& p_{5, l \psi}^{h}=p^{h}\left(\beta_{\eta_{l \psi}}^{\uparrow}, \alpha_{\theta_{l \psi}}^{\uparrow}, z_{k_{l \psi}}^{\downarrow}\right) ; \\
& p_{6, l \psi}^{h}=p^{h}\left(\beta_{\eta_{l \psi}}^{\downarrow}, \alpha_{\theta_{l \psi}}^{\uparrow}, \dot{z}_{k_{l \psi}}^{\downarrow}\right) ; \\
& p_{7, l \psi}^{h}=p^{h}\left(\beta_{\eta_{l \psi}}^{\uparrow}, \alpha_{\theta_{l \psi}}^{\downarrow}, \dot{z}_{k_{l \psi}}^{\downarrow}\right) ; \\
& p_{8, l \psi}^{h}=p^{h}\left(\beta_{\eta_{l \psi}}^{\downarrow}, \alpha_{\theta_{l \psi}}^{\downarrow}, \dot{z}_{k_{l \psi}}^{\downarrow}\right) \text {, }
\end{aligned}
$$

where the sign $\downarrow$ means the previous, and the sign $\uparrow$ the next value of the given parameter of the physically performed projection, as specified by (4), (5) or (6).

The trilinear interpolation mentioned above processes measurements specified by (9) as follows

$$
\dot{p}^{h}\left(\beta_{l \psi}, \alpha_{l \psi}^{h}, \dot{z}_{l \psi}\right)=\sum_{n=1}^{8} c_{n, l \psi} p_{n, l \psi}^{h}
$$

where the coefficients of interpolation $c_{n, l \psi}$ can be established in the following way

$$
c_{n, l \psi}=\left(1-\frac{|\Delta \beta|}{\Delta_{\eta}}\right) \cdot\left(1-\frac{|\Delta \theta|}{\Delta_{\theta}}\right) \cdot\left(1-\frac{|\Delta \dot{z}|}{\Delta_{\dot{z}_{k}}}\right),
$$

where $|\Delta \theta|,|\Delta \theta|$ and $|\Delta \dot{z}|$ are the differences between the parameters of one of the chosen projections $p_{n, l \psi}^{h}$ and the parameters specified by relations (4), (5) and (6), for dimensions $\beta, \alpha^{h}$ and $\dot{z}$, respectively.

It is worth noting that the interpolated ray passes through the various tissues along a path longer than that of the approximated parallel ray. 
We therefore have to make a correction for this effect. This correction can be performed by multiplying the interpolated projection by the following factor

$$
\begin{aligned}
& \operatorname{CORR}_{1}= \\
& =\frac{\left(\mathrm{R}_{f}+\mathrm{R}_{d}\right) \cos \left(\alpha^{h}-\alpha_{p}^{h}-\alpha_{\psi}^{p}\right) \cos v}{\sqrt{w^{2}+v^{2}+\left(\mathrm{R}_{f}+\mathrm{R}_{d}\right)^{2}} \cdot \sqrt{\sin ^{2} \alpha_{\psi}^{p}+\cos ^{2} v \sin ^{2} \alpha_{\psi}^{p}}}+ \\
& \frac{w \sin \left(\alpha^{h}-\alpha_{p}^{h}-\alpha_{\psi}^{p}\right) \cos v-v \sin \alpha_{\psi}^{p} \sin v}{\sqrt{w^{2}+v^{2}+\left(\mathrm{R}_{f}+\mathrm{R}_{d}\right)^{2}} \cdot \sqrt{\sin ^{2} \alpha_{\psi}^{p}+\cos ^{2} v \sin ^{2} \alpha_{\psi}^{p}}},
\end{aligned}
$$

where Equations (7) and (8) still hold true.

To avoid having to transfer the results of the reconstruction procedure from the local coordinate system of the reconstructed cross-section to the global coordinate system, the projection values should be corrected by a second factor, which can be expressed in the following way

$$
\operatorname{COR}_{2}=\frac{\cos v}{\sqrt{\cos ^{2} \alpha^{p}+\cos ^{2} v \sin ^{2} \alpha_{\psi}^{p}}} .
$$

Taking all the above considerations together, it is possible to write the approximated parallel projection as

$$
p^{p}\left(s_{l}, \alpha_{\psi}^{p}\right)=\dot{p}^{h}\left(\beta_{l \psi}, \alpha_{l \psi}^{h}, \dot{z}_{l \psi}\right) \cdot \operatorname{CORR}_{1} \cdot \operatorname{COR}_{2}
$$

where $\dot{p}^{h}\left(\beta_{l \psi}, \alpha_{l \psi}^{h}, \dot{z}_{l \psi}\right)$ is an interpolated value of the helical projection specified by relation (10).

After the operations described in this Section, we have a set of the parallel projections $p^{p}\left(s_{l}, \alpha_{\psi}^{p}\right)$ ready to be applied to a reconstruction method designed for this type of scanner geometry.

\subsection{Statistical reconstruction procedure}

As we already have the parallel projections prepared, as shown in previous subsections, we can now apply our original approach to the reconstruction problem. This approach has been presented previously in different forms for parallelbeam [12, 13, 20], fan-beam [14] and cone-beam [15] scanners.

A rectangle in Figure 1 marks the part of the algorithm that is processed using parallel beams (in this case, virtual parallel beams). The individual operations which are included in this fundamental part of our algorithm are presented below.

\subsubsection{Back-projection operation}

The first step in this part of our algorithm is a back-projection operation. This operation can be written mathematically as

$$
\tilde{\mu}(i, j)=\Delta_{\alpha^{h}} \sum_{\psi} \dot{p}^{p}\left(s_{i j}, \alpha_{\psi}^{p}\right),
$$

where: $s_{i j}=i \Delta_{s} \cos \alpha_{\psi}^{p}+j \Delta_{s} \sin \alpha_{\psi}^{p}$ is the coordinate defining the position of point $(i, j)$ on the screen, during a projection carried out at angle $\alpha_{\psi}^{p}$; $\Delta_{s}=\Delta_{x y}$ is the distance between pixels in the reconstructed image.

It is necessary to use an interpolation to evaluate projections at points $s_{i j}$ based on the virtual projections $p^{p}\left(s_{l}, \alpha_{\psi}^{p}\right)$. We can obtain an approximation of the projection $p^{p}\left(s_{l}, \alpha_{\psi}^{p}\right)$ expressed by

$$
\begin{aligned}
& \dot{p}^{p}\left(s_{i j}, \alpha_{\psi}^{p}\right)= \\
& \quad \Delta_{s} \sum_{l} p^{p}\left(l \Delta_{s}, \alpha_{\psi}^{p}\right) \cdot \overline{i n t}\left(s_{i j}-l \Delta_{s}\right),
\end{aligned}
$$

where $\operatorname{int}^{-}(\Delta s)$ is an interpolation function.

In the simplest case, linear interpolation can be used, and the interpolation can be formulated in the following way

$$
\dot{p}^{p}\left(s_{i j}, \alpha_{\psi}^{p}\right)=\sum_{m=1}^{2} c_{m, i j} p_{m, i j}^{p},
$$

where coefficients of interpolation $c_{m, l \psi}$ can be established as follows

$$
c_{m, i j}=\left(1-\frac{|\Delta s|}{\Delta_{s}}\right),
$$

where $|\Delta s|$ is the difference between parameter $s_{i j}=$ $i \Delta_{s} \cos \alpha_{\psi}^{p}+j \Delta_{s} \sin \alpha_{\psi}^{p}$ and one of the chosen projections $p_{m, l \psi}^{p}$, which are established as follows

$$
p_{1, l \psi}^{p}=p^{p}\left(\Delta_{s} l^{\uparrow}, \alpha_{l \psi}^{p}\right) ; \quad p_{2, l \psi}^{p}=p^{p}\left(\Delta_{s} l^{\downarrow}, \alpha_{l \psi}^{p}\right) .
$$

It is very important at this moment to take into consideration the statistical nature of the image $\tilde{\mu}(i, j)$ obtained as presented above.

Let us start from the statistical nature of the measurements used in computed tomography by taking into account the following definition

$$
p \equiv \ln \left(\frac{I(0)}{I}\right)=\ln \left(\frac{n_{0}}{n}\right),
$$


where: $I(0)$ is the initial X-ray intensity (determined during the calibration of the CT scanner); $I$ is the X-ray intensity detected after passing through a body; $n_{0}$ is the initial photon count; $n$ is the photon count registered by an X-ray detector.

We can assume that $n$ is the realization of a Poisson-distributed random variable, i.e. $N$, with an expected value $n^{*}$.

Next, we assume that $P$ is a random variable that represents the stochastic nature of the projection value $p$ established by measurement. Taking into account the definition (20), we can treat variable $P$ as a function of the random variable $N$, as follows

$$
P=\ln \left(\frac{n_{0}}{N}\right) .
$$

In our case, the projection values $p$ in (20) are represented physically by $p^{h}\left(\beta_{\gamma}, \alpha_{\theta}^{h}, \dot{z}_{k}\right)$.

In our further considerations, it will be assumed that the variances $\sigma_{\eta \theta k}^{2}$ of the variances $P$ are estimated by $\frac{1}{n_{\eta \theta k}}=\frac{\mathrm{e}^{p^{h}\left(\beta_{\eta}, \alpha_{\theta}^{h}, \dot{z}_{k}\right)}}{n_{0}}$ (see e.g. [21]). Bearing in mind Eqs (10), (13), (12), (17), we can notice that the random variable representing the virtual parallel projections $p^{p}\left(l \Delta_{s}, \alpha_{\psi}^{p}\right)$ is a linear function of the random variable $P$. Therefore, it is possible to estimate the variance in the following way

$$
\begin{aligned}
& \sigma_{i j, \psi}^{2} \cong \sum_{m=1}^{2} c_{m, i j}^{2} . \\
& . \operatorname{CORR}_{1, l \psi}^{2} \cdot \operatorname{CORR}_{2, l \psi}^{2} \sum_{n=1}^{8} c_{n, l \psi}^{2} \frac{\mathrm{e}^{p_{n, l \psi}^{h}}}{n_{0}}
\end{aligned}
$$

The above considerations describe the statistical nature of only one interpolated value which is obtained at a given projection angle $\alpha_{\psi}$. Of course, it is necessary to take into account all approximated parallel projections $p^{p}\left(s_{i j}, \boldsymbol{\alpha}_{\psi}^{p}\right)$ used by the backprojection operation. This means that, bearing in mind relation (15) the following summation is obtained

$$
\begin{aligned}
& \tilde{\mu}(i, j)=\Delta_{\alpha^{p}} \sum_{\psi} \sum_{m=1}^{2} c_{m, i j} \cdot \\
& \cdot \operatorname{CORR}_{1, l \psi}^{2} \cdot \operatorname{CORR}_{2, l \psi}^{2} \sum_{n=1}^{8} c_{n, l \psi} p_{n, l \psi}^{h} .
\end{aligned}
$$

Taking into account the fact that in the statistical case considered here, an approximate analytical re- construction problem in the form of a shift invariant system (see e.g. [12]) involves expected values of attenuation coefficients, i.e. $\mu^{*}(i, j)$, we can write as follows

$$
\tilde{\mu}^{*}(i, j) \cong\left(\Delta_{s}\right)^{2} \sum_{\bar{i}} \sum_{\bar{j}} \mu^{*}(\bar{i}, \bar{j}) h_{\Delta i, \Delta j}
$$

where

$$
\begin{aligned}
& h_{\Delta i, \Delta j}=\Delta_{\alpha} \\
& \quad \sum_{\psi=0}^{\Psi-1}\left(1-\left|i \cos \psi \Delta_{\alpha^{p}}+j \sin \psi \Delta_{\alpha^{p}}\right|\right) .
\end{aligned}
$$

Let us assume that all the projections $\dot{p}^{p}\left(s_{i j}, \alpha_{\psi}^{p}\right)$ from (17) are realizations of the independent random variables $P_{i j, \psi}$, for a specific pixel $(i, j)$. According to the Lyapunov central limit theorem, the distribution of the sum $P_{\Sigma}(i, j)=\sum_{\psi=0}^{\Psi-1} P_{i j, \psi}$ converges to that of a normal random variable, as $\Psi$ goes to infinity. Therefore, if the number of projections $\Psi$ is sufficiently large then the random variable $P_{\Sigma}(i, j)$ tends to the Gaussian distribution function, as follows

$$
\begin{array}{r}
f_{P r_{i j}}(\tilde{\mu}(i, j))=\frac{1}{\sqrt{2 \pi} \sigma_{\Sigma}(i, j)} \\
\quad \exp \left(-\frac{\left(\tilde{\mu}(i, j)-\tilde{\mu}^{*}(i, j)\right)^{2}}{2 \sigma_{\Sigma}^{2}(i, j)}\right),
\end{array}
$$

where

$$
\sigma_{\Sigma}(i, j)=\sqrt{\sigma_{1}^{2}(i, j)+\sigma_{2}^{2}(i, j)+\cdots+\sigma_{\Psi-1}^{2}(i, j)},
$$

where $\sigma_{\psi}^{2}(i, j)$ are the variances of the random variables $P_{i j, \psi}$ described by (22), and $\tilde{\mu}^{*}(i, j)$ is the expected value of the random variable $P_{\Sigma}(i, j)$.

The probability function (26) considers only one pixel $(i, j)$ in the reconstructed image. It is necessary to extend this function to the function $f_{P r}(\tilde{\mu})$, if all pixels in this image have to be taken into account, as follows

$$
\bigwedge_{\tilde{\mu}} f_{P r}(\tilde{\mu})=\prod_{i=1}^{\mathrm{I}} \prod_{j=1}^{\mathrm{I}} f_{P r_{i j}}(\tilde{\mu}(i, j)) .
$$

The probability function expressed by (28) forms the basis for the formulation of the objective of an appropriately formulated optimization problem. 


\subsubsection{Iterative reconstruction procedure}

As was mentioned in sec. 2.4.1, the reconstruction problem expressed by relation (28) will be used to reformulate as an optimization problem. It is very convenient in this case to use the maximum likelihood (ML) method, applying the log form of (28) as the objective for this strategy, as follows

$$
\begin{aligned}
L 1= & \ln \left(f_{\operatorname{Pr}}(\tilde{\mu})\right)= \\
& \sum_{i=1}^{\mathrm{I}} \sum_{j=1}^{\mathrm{I}}\left(b-\frac{\left(\tilde{\mu}(i, j)-\tilde{\mu}^{*}(i, j)\right)^{2}}{2 \sigma_{\Sigma}^{2}(i, j)}\right),
\end{aligned}
$$

where $\sigma_{\Sigma}^{2}(i, j)$ is expressed by (27), and constant $b=-\ln \left(\sqrt{2 \pi} \sigma_{\Sigma}(i, j)\right)$. Because $b$ is constant, we neglect this term when using the gradient method during the optimization procedure, giving the next form of the objective

$$
L 2=-\sum_{i=1}^{\mathrm{I}} \sum_{j=1}^{\mathrm{I}}\left(\frac{1}{2 \sigma_{\Sigma}^{2}(i, j)}\left(\tilde{\mu}^{*}(i, j)-\tilde{\mu}(i, j)\right)^{2}\right) .
$$

The main idea of algorithms based on the maximum likelihood (ML) method is to estimate the expected value of the reconstructed image at particular pixels (see e.g. [4]). Then, our reconstruction problem can be formulated as an optimization procedure, as follows

$$
\mu_{\min }=\arg \min _{\mu} \frac{1}{2} \sum_{i=1}^{\mathrm{I}} \sum_{j=1}^{\mathrm{J}} \frac{1}{\sigma_{\Sigma}^{2}(i, j)}(e(i, j))^{2},
$$

where

$$
e(\bar{i}, \bar{j})=\sum_{i} \sum_{j} \mu(i, j) \cdot h_{\Delta i, \Delta j}-\tilde{\mu}(\bar{i}, \bar{j}) .
$$

Of course, value $\tilde{\mu}$ in Eq. (32) represents the image obtained after the back-projection operation, $h_{\Delta i, \Delta j}$ are constant coefficients of the shift invariant system, and $\mu$ represents an estimation of the expected values of the reconstructed image.

However, results obtained earlier (see e.g. [11]) show that the reconstruction problem (31) is better conditioned than methods based on the D-D model. In any case, it needs to be regularized in some way, often by the introduction of an additional regularization term. This term relates to the maximum $a$ priori (MAP) estimate framework. This Bayesian framework can be expressed as follows

$$
\begin{aligned}
\mu_{\min } & =\arg \min _{\mu} \\
& a_{1} \frac{1}{2} \sum_{i=1}^{\mathrm{I}} \sum_{j=1}^{\mathrm{J}} \frac{1}{\sigma_{\Sigma}^{2}(i, j)}(e(i, j))^{2}+a_{2} f(\mu),
\end{aligned}
$$

where $f(\mu)$ is a regularization term; $a_{1}$ and $a_{2}$ are constant coefficients. The regularization term may take different forms, for instance, the total variation (TV) regularization [23].

Therefore, we can postulate that it would be possible to find the optimal image $\mu^{*}$, that is the estimation of the expected values of the reconstructed image $\mu$, using the optimization algorithm, as expressed by (31). In our case, the gradient descent method was chosen to search for the optimal solution. The iterative expression based on this strategy takes the following form

$$
\begin{aligned}
& \mu^{(t+1)}(i, j)=\mu^{(t)}(i, j)- \\
& \quad a_{1} \sum_{\bar{i}} \sum_{\bar{j}} e^{(t)}\left(x_{\bar{i}}, y_{\bar{j}}\right) h_{\Delta i, \Delta j} a_{2} f^{\prime}(\mu),
\end{aligned}
$$

$f^{\prime}(\mu)$ is the derivative of the regularizing function (for the TV approach see [22]), and

$$
e^{(t)}(\bar{i}, \bar{j})=\sum_{i} \sum_{j} \mu^{*(t)}(i, j) \cdot h_{\Delta i, \Delta j}-\tilde{\mu}(\bar{i}, \bar{j}) .
$$

In the method presented here, every iteration of the reconstruction procedure is comprised of two steps, as described by relations (34) and (35). However, the convolutions presented in these relations can be changed into multiplications if it is transformed into the frequency domain. Thanks to this, the computational complexity is decreased from an $I^{4}$ to a $\log _{2} I^{2}$ problem by using FFT and IFFT algorithms.

\section{Experimental Results}

In experiments, which were carried out, the well-known 3D Shepp-Logan mathematical phantom of the head was used (see e.g. [24]). However, 
in order to facilitate the calculations, all the values of the attenuation coefficients used in the model were divided by a factor of $10^{-3}$. Next, the model modified in this way was used to generate projections with a Poisson probability distribution. After that, the pitch (at $\lambda=2$ ) was determined, and then those positions on the $z$ axis at which the planes of the reconstruction intersect the axis were chosen $\left(z_{p}=0, z_{p}=-117\right.$ and $\left.z_{p}=325\right)$. For each position $z_{p}$, the angle $\gamma$ was calculated according to the formula (3).

For parallel projections, there was a fixed number $(L=1024)$ of virtual detectors on the screen. The number of parallel views was chosen as $\Psi=$ 1610 per half-rotation, and the size of the processed image was fixed at $\mathrm{I} \times \mathrm{I}=1024 \times 1024$ pixels. On the other hand, for spiral cone beam geometry, the resolution of the measurements performed was defined as follows: $\Delta_{\theta}=\pi / 1609, \Delta_{\eta}=\pi / 3000, \Delta_{k}=$ 0.25 .

After that, the back-projection operation can be performed using the method, which is described in Section 2.3, and then the iterative reconstruction procedure, which is explained in Section 2.4.

It is worth underlining that the coefficients $h_{\Delta i, \Delta j}$, necessary for the iterative procedure, can be pre-calculated, i.e. established before the reconstruction process is started.

Having determined the coefficients $h_{\Delta i, \Delta j}$, it is then possible to start the iterative reconstruction procedure. The image obtained after the backprojection operation was subjected to a process of reconstruction using a gradient descent method.

The evaluation of the quality of the reconstructed image was performed using an error measure defined as follows

$$
M S E=\frac{1}{I^{2}} \sum_{i=1}^{\mathrm{I}} \sum_{j=1}^{\mathrm{J}}\left(\mu^{(t)}(i, j)-\mu(i, j)\right)^{2},
$$

where: $\mu^{(t)}(i, j)$ is the reconstructed image after $t$ iterations and $\mu(i, j)$ is the original image of the artificial 3D Shepp-Logan phantom.

Views of the results obtained, i.e. reconstructed images of the mathematical phantom in cross-section after $t=5000$ iterations, are presented in Table 1.C. For comparison, the original phan- tom images are shown in Table 1.A and images reconstructed using the traditional ASSR method are shown in Table 1.B. It is worth noting that the starting point of this iterative reconstruction procedure can be a reconstructed image using any standard reconstruction method. In our computer simulations, the traditional ASSR algorithm was used (projections were convoluted with the Shepp-Logan kernel).

To evaluate the proposed statistical modelbased reconstruction method in real conditions, we have used measurements obtained on a commercial scanner to conduct the reconstruction procedure presented in this paper, and for comparison, we have used results obtained using a referential approach, i.e. the traditional ASSR algorithm. A comparison of images obtained using the traditional ASSR method and those using the ASSR approach with the iterative statistical reconstruction procedure is presented in Figure 7 (measurements were carried out using a cone-beam helical scanner: Somatom Definition AS+, Siemens Healthcare, Forchheim, Germany). Results of the experiments performed using projections obtained on the Somatom Definition AS+ scanner, Siemens Healthcare, Forchheim, Germany, $100 \mathrm{kVp}$ and effective $200 \mathrm{mAs}$, 70s after iodinated contrast injection, $R_{f d}=1085.6 \mathrm{~mm}, \mathrm{R}_{f}=595 \mathrm{~mm}$, number of views per rotation $\Psi=1152$, number of pixels in detector panel 736, detector dimensions were $1.09 \mathrm{~mm} \times 1.28 \mathrm{~mm}$. 
Table 1. View of the images $\left(\left(C=1.05 \cdot 10^{-3}, W=0.1 \cdot 10^{-3}\right)\right)$ : original images $\left(c_{2}=10^{-4}, \beta=10^{-10}\right)$ (A); reconstructed images obtained using the ASSR method (B); reconstructed images obtained using the statistical iterative procedure proposed in this paper (after 3000 iterations) (C)

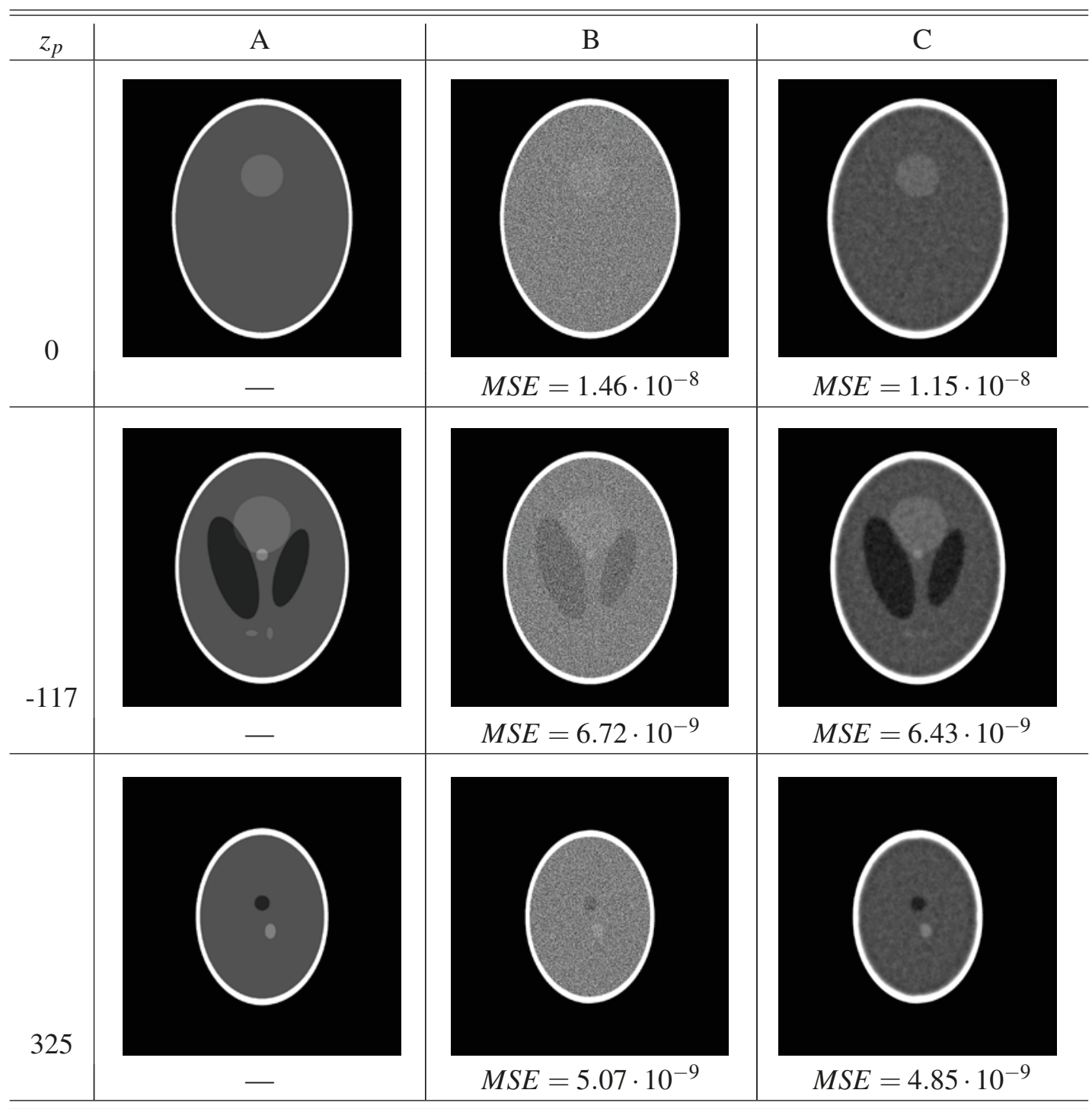


(A)

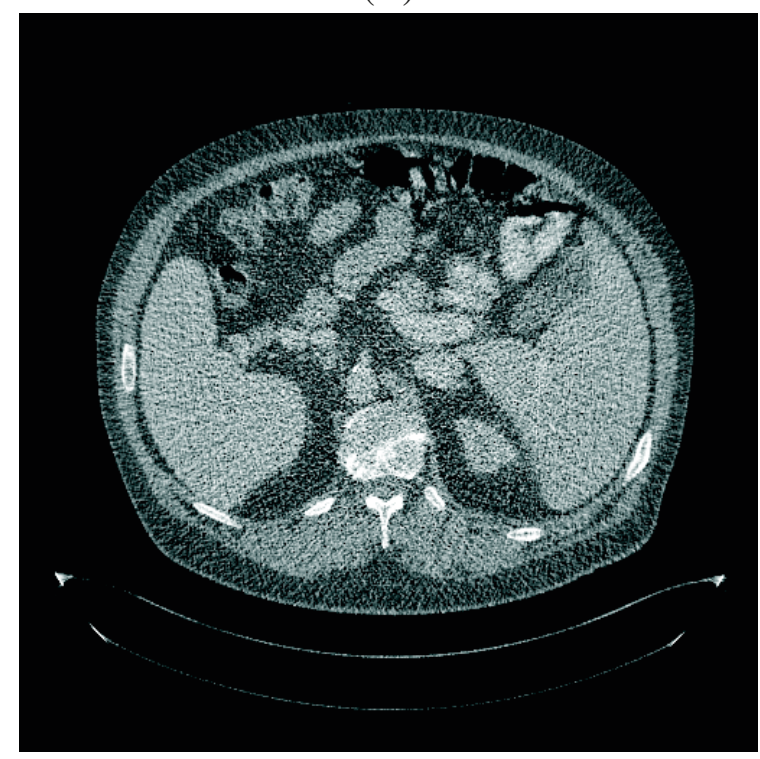

(B)

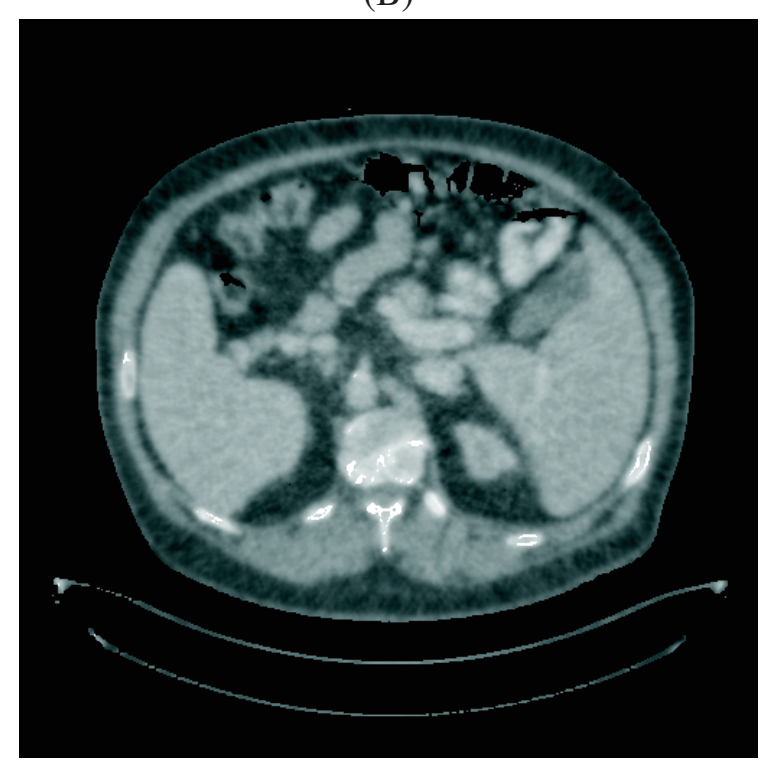

Figure 7. Image reconstructed using a traditional filtration/back-projection algorithm, i.e. the ASSR

(A); Image reconstructed using the method proposed in this paper, which is based on the $\mathrm{C}-\mathrm{C}$ statistical formulation of the reconstruction problem and on the ASSR method (after 10000 iterations)

\section{Conclusion}

In this paper, it has been proven that the proposed practical statistical approach can be applied to a spiral scanner. Simulations have shown, that this reconstruction method is very fast (thanks to the use of FFT algorithms) and gives satisfactory results with suppressed noise. Thanks to the use of the $\mathrm{C}-\mathrm{C}$ model in the proposed approach, it is possible to avoid the very serious drawbacks present in reconstruction approaches utilizing the D-D model. These are particularly noticeable in reconstruction algorithms for spiral scanners. First of all, the computational complexity for $2 \mathrm{D}$ reconstruction geometry (e.g. parallel rays) is proportional to $I^{2} \Psi \times N$ for each iteration, for approaches based on the D-D model. Our original approach only needs approximately $4 I^{2} \log _{2}(2 I)$ operations. For the $3 \mathrm{D}$ reconstruction problem these proportions change to $I^{2} \times$ numbers_of_reconstructed_crosssections $\times \Psi \times N$, while our approach still only requires $4 I^{2} \log _{2}(2 I)$ i.e. there is no increase. It is worth noting that in both algorithms, backprojection is performed. Of course, the number of iterations which need to be performed to produce an image of satisfactory quality is greater than in the traditional ASSR algorithm. This means that our method is more computationally demanding than the traditional equivalent. Note, however, that if we consider an image of $1024 \times 1024$ pixels and a suitable value of $\Psi=1069$ we have to perform about 10000 iterations. Moreover, elements of the model matrix $h_{\Delta i, \Delta j}$ can be pre-calculated before the reconstruction procedure is started, and these are the same for all pixels of the reconstructed image. The images of the cross-sections of the mathematical phantom investigated were reconstructed with high accuracy when compared with the standard ASSR method. This was measured objectively using a quality measure which takes into consideration the statistical nature of the projections.

\section{Acknowledgement}

The authors thank Dr. Cynthia McCoullough and the American Association of Physicists in Medicine for providing the Low-Dose CT Grand Challenge dataset.

This work was partly supported by The National Centre for Research and Development in Poland (Research Project POIR.01.01.01-000463/17). 


\section{References}

[1] G. N. Ramachandran, A. V. Lakshminarayanan, Three-dimensional reconstruction from radiographs and electron micrographs: II. Application of convolutions instead of Fourier transforms, Proc. Nat. Acad. Sci. of USA, vol. 68, 1971, pp. 2236-2240.

[2] R. M. Lewitt, Reconstruction algorithms: transform methods, Proc. of the IEEE, vol. 71, 1983, pp. 390-408.

[3] J. D. Mathews et al., Cancer risk in 680 peope expose to computed tomography scans in childhood or adolescent: data inkage study of 11 million Australians, British Medical Journal, f2360, 2013, pp. 346-360

[4] K. Sauer, C. Bouman, A local update strategy for iterative reconstruction from projections, IEEE Tran. Signal Proc., vol. 41, 1993, pp. 534-548.

[5] C. A. Bouman, K. Sauer, A unified approach to statistical tomography using coordinate descent optimization, IEEE Tran. Image Proc., vol. 5, 1996, pp. 480-492.

[6] Ding Q., Long Y., Zhang X., Fessler J.A.: Modeling mixed Poisson-Gaussian noise in statistical image reconstruction for $\mathrm{x}$-ray CT. In: Proc. of the 4th International Conference on Image Formation in X-Ray Computed Tomography, Bamberg, Germany, 399-402 (2016)

[7] Geyer, L.L., et al.: State of the art: iterative CT reconstruction techniques. Radiology 276, 339-357 (2017)

[8] J. -B Thibault, K. D. Sauer, C. A. Bouman, J. Hsieh, A three-dimensional statistical approach to improved image quality for multislice helical CT, Med. Phys., vol. 34, 2007, pp. 4526-4544.

[9] B. DeMan, S. Basu, Distance-driven projection and backprojection in three dimensions, Phys. Med. Biol., vol. 49, 2004, pp. 2463-2475.

[10] Y. Zhou, J.-B Thibault, C.A. Bouman, J. Hsieh, K.D. Sauer, Fast model-based X-ray CT reconstruction using spatially non-homogeneous ICD optimization, IEEE Tran. Image Proc., vol. 20, 2011, pp. 161-175.

[11] R. Cierniak, A. Lorent, Comparison of algebraic and analytical approaches to the formulation of the statistical model-based reconstruction problem for x-ray computed tomography, Computerized Medical Imaging and Graphics, vol. 52, 2016, pp. 19-27.
[12] R. Cierniak, A new approach to tomographic image reconstruction using a Hopfield-type neural network, International Journal Artificial Intelligence in Medicine, vol. 43, 2008, pp. 113-125.

[13] R. Cierniak, A new approach to image reconstruction from projections problem using a recurrent neural network, International Journal of Applied Mathematics and Computer Science, vol. 183, 2008, pp. 147-157.

[14] R. Cierniak, New neural network algorithm for image reconstruction from fan-beam projections, Neurocomputing, vol. 72, 2009, pp. 3238-3244.

[15] R. Cierniak, A three-dimensional neural network based approach to the image reconstruction from projections problem, Lecture Notes in Artificial Intelligence, 6113, 2010, pp.505-514.

[16] F. Noo, M. Defrise, R. Clackdoyle, Single-slice rebinning method for helical cone-beam CT, Phys. Med. Biol., vol. 44, 1999, pp. 561-570.

[17] H. Bruder, M. Kachelrieß, S. Schaller, K. Stierstorfer, T. Flohr, Single-slice rebinning reconstruction in spiral cone-beam computed tomography, IEEE Trans. Med. Imag., vol. 9, 2000, pp. 873-887.

[18] M. Kachelrieß, S. Schaller, W. A. Kalender, Advanced single-slice rebinning in cone-beam spiral CT, Med. Phys., vol. 27, 2000, pp.754-773.

[19] M. Kachelrieß; T. Fuchs, S. Schaller, et al, Advanced single-slice rebinning for tilted spiral conebeam CT, Med. Phys., vol. 28, 2001, pp.10331041.

[20] R. Cierniak, A novel approach to image reconstruction from projections using Hopfield-type neural network, Lecture Notes in Artificial Intelligence, 4029, 2006, pp. 890-898.

[21] J. -B. Thibault, C. A. Bouman, K. D. Sauer, J. Hsieh, A recursive filter noise reduction in statistical iterative tomographic imaging, Proc. of SPIE-IS\&T Symposium on Electronic Imaging Science and Technology-Computational Imaging, vol. 6065, 2006, pp. 15-19.

[22] J. -F. Aujol, Some first-order algorithms for total variation based image restoration, J. Math. Im. Vision, vol. 34, 2009, pp. 307-327.

[23] L.I. Rudin, S. Osher, E. Fatemi, Nonlinear total variation based noise removal algorithms, Physica D: Nonlinear Phenomena, vol. 60, 1992, pp. 259268.

[24] A. C. Kak, M. Slanley, Principles of computerized tomographic imaging, IEEE Press, New York, 1988. 


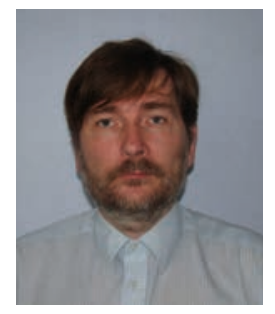

Robert Cierniak received an M.S. degree in Electronics Engineering in 1990, and a Ph.D. degree in Computer Science in 1995, both from Cracow University of Technology, Poland. From 1991 to 2008 he conducted research at the Czestochowa University of Technology, Poland. He spent the period from 1991 to 1996 with the Institute of Electronics and Control Systems, and the period from 1996 to 2009 with the Institute of Computer Engineering, as an assistant professor. He has now become a full professor at this Institute. His main interests include neural networks, biomedical applications of artificial intelligence, computer vision and image processing.

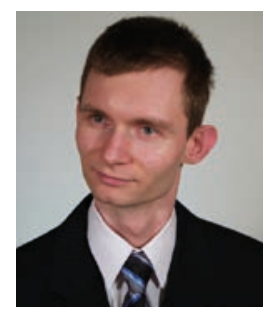

Piotr Pluta was born in Częstochowa, Poland, in 1988. He received the M.Sc. degree in computer science from Universitas Opoliensis, Poland, in 2013. Currently, he is a Ph.D. student in computer science at the Department of Intelligent Computer Systems at Czestochowa University of Technology. His research interests include image reconstruction from projections.

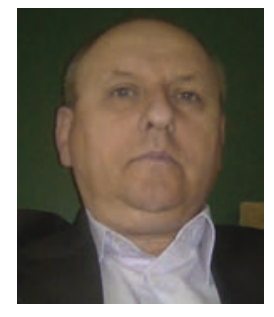

Andrzej Kaźmierczak is an assistant professor at the University of Social Sciences in Łódź. He received the M.Sc. degree in theoretical physics from University of Lodz, Poland and the Ph.D. degree in natural sciences from the Medical University in Lodz, Poland. His research interest include earth geopotential, geodynamics, and numerical computer simulations. 